\title{
Combined Transcriptome and Metabolome Integrated Analysis of Acer mandshuricum to Reveal Candidate Genes Involved in Anthocyanin Accumulation
}

Shikai Zhang

Northeast Forestry University

Wang Zhan

Baishishan Forestry Bureau

Anran Sun

Northeast Forestry University

Ying Xie

Northeast Forestry University

Zhiming Han

Northeast Forestry University

Xibin Qu

Baishishan Forestry Bureau

Jiayi Wang

Baishishan Forestry Bureau

Laifu Zhang

Baishishan Forestry Bureau

Mingshun Tian

Baishishan Forestry Bureau

Xuhong Pang

Baishishan Forestry Bureau

Jinbao Zhang

Baishishan Forestry Bureau

Xiyang Zhao ( $\nabla$ zhaoxyphd@163.com )

Northeast Forestry University

\section{Research Article}

Keywords: 3-0-glucoside, A. mandshuricum

Posted Date: August 13th, 2021 
DOI: https://doi.org/10.21203/rs.3.rs-796758/v1

License: (c) (1) This work is licensed under a Creative Commons Attribution 4.0 International License. Read Full License

Version of Record: A version of this preprint was published at Scientific Reports on November 30th, 2021. See the published version at https://doi.org/10.1038/s41598-021-02607-2. 


\section{Abstract}

The red color formation of Acer mandshuricum leaves is caused by the accumulation of anthocyanins primarily, but the molecular mechanism researches which underlie anthocyanin biosynthesis in $A$. mandshuricum were still lacking. Therefore, we combined the transcriptome and metabolome and analyzed the regulatory mechanism and accumulation pattern of anthocyanins in leaf color change periods in three different leaf color states. In our results, 26 anthocyanins were identified. Notably, the metabolite cyanidin 3-0-glucoside was found that significantly correlated with the color formation, was the predominant metabolite in anthocyanin biosynthesis of $A$. mandshuricum. By the way, two key structural genes ANS (Cluster-20561.86285) and BZ1 (Cluster-20561.99238) in anthocyanidin biosynthesis pathway were significantly up-regulated in $\mathrm{RL}$, suggesting that they might enhance accumulation of cyanidin 3-0-glucoside which is their downstream metabolite, and contributed the red formation of A. mandshuricum leaves. Additionally, most TFs (e.g., MYBs, bZIPs and bHLHs) were detected differentially expressed in three leaf color stages that could participate in anthocyanin accumulation. This study sheds light on the anthocyanin molecular regulation of anthocyanidin biosynthesis and accumulation underlying the different leaf color change periods in $A$. mandshuricum, and it could provide basic theory and new insight for the leaf color related genetic improvement of $A$. mandshuricum.

\section{Introduction}

Acer mandshuricum, a precious colored-leaf tree in landscapes, belongs to the genus Acer (Aceraceae) and is mainly distributed in northeastern China1. Owing to its great ornamental value, A. mandshuricum has been selected as the ideal tree species for urban landscaping. It has a visually appealing tree type, and the leaves can change from green to very bright pink or fuchsia in autumn (Fig. 1)2. With the development of the world economy, the process of urbanization is accelerating, and people's demands on the urban environment have been gradually increasing. Obviously, colored-leaf plants can play an important role in the landscape due to their colorful leaves, strong resistance and ease of cultivation. A large number of $A$. mandshuricum trees have been transplanted into urban landscaping3. However, some problems have also appeared. For example, the color change period was greatly shortened when wild $A$. mandshuricum was transplanted to the city. Moreover, it was found that some genes related to colored leaves in natural forest trees could not be passed on steadily to future generations. In view of the important economic and ornamental value of $A$. mandshuricum, it is an urgent problem to study the Anthocyanidin accumulation mechanism for color-related genetic improvement.

Numerous scientific studies have shown that changes in the color of leaves are caused by changes in the pigment content of their bodies, including chlorophyll, lutein, carotene, anthocyanin and more4-6. Among the many pigments, anthocyanins have received much attention because of their considerable ability to control leaf color changes7. Anthocyanin is a kind of water-soluble natural pigment widely found in plants. There are more than 20 kinds of known anthocyanins, 6 of which are more common in plants: pelargonidin, cyanidin, delphinidin, peonidin, petunidin and malvidin8-10. The amount of anthocyanins 
present can directly affect the color change of plants. At present, the synthetic pathway of anthocyanins has been elucidated in many studies, and it is a branch of the phenylpropanoid biosynthetic pathway 11,12 . The molecular mechanism of key structural genes in its pathways, such as phenylalanine ammonia-lyase ( $P A L)$, 4-coumarate-CoA ligase ( $4 C L)$, chalcone synthase $(C H S)$, chalcone isomerase $(C H I)$, dihydroflavonol 4-reductase (DFR), anthocyanin synthase (ANS) and more, has been clarified13-15. At the same time, the biosynthesis of anthocyanins is also coregulated by some transcription factors, among which the greatest influences were MYBs, bHLHs, bZIPs and so on 16,17. Therefore, research on the biosynthesis process of anthocyanins in A. mandshuricum will help people to better interpret the molecular mechanism of leaf color change and will also contribute to genetic improvement and germplasm innovation.

In recent years, advanced sequencing technology has developed rapidly, and multi-omics research has become the main means for people to explore unknown areas of cells18,19. Correlation analysis of the transcriptome and metabolome can realize the co-expression analysis of differential genes and differential metabolites in timing expression20-22. Exploring the causal relationship between genes and metabolites and combining biological function analysis such as functional annotation and enrichment of metabolic pathways is important to identify the key metabolic pathways, key genes and key metabolites23-25. Thus, the relationship between plant regulation mechanisms and biometric function can be analyzed systematically.

At present, the systematic study of leaf color changes and the precise positioning of leaf color-related genes in A. mandshuricum have not been reported. In this study, we profiled the transcriptome and metabolome changes in $A$. mandshuricum during various leaf color change stages to investigate the coloration mechanism. Our results shed light on the candidate genes and metabolic pathways underlying the leaf color change in $A$. mandshuricum and could provide fundamental theory and novel insight for the genetic improvement of $A$. mandshuricum.

\section{Results}

Metabolome profiling in different color period samples. Throughout the leaf color change cycle of $A$. mandshuricum, it undergoes a transition from green leaves $(\mathrm{GL})$ to half red leaves (HRL) and then to red leaves (RL) (Fig. 1). Therefore, to detect the change in metabolite concentration (mainly focused on anthocyanins) associated with the leaf color change process in A. mandshuricum, three types of leaves during different leaf color change periods were used for experiments. We profiled the metabolome of the three samples using UPLC (ultraperformance liquid chromatography) and a widely targeted metabolomics approach. Then, 26 compounds involving anthocyanins were detected and grouped into 8 classes (Table S1 and Fig. 2A), including 5 cyanidins, 4 pelargonidins, 4 peonidins, 4 malvidins, 4 proanthocya-nidins, 2 delphinidins, 2 flavonols and 1 petunidin. Subsequently, the metabolite concentration data were used to perform a hier-archical heatmap clustering analysis of those samples. Among them, most metabolites, such as cyanidins (cyanidin 3,5-0-diglucoside, cyanidin 3-0-(6-0-malonylbeta-D-glucoside), cyanidin 3-0-arabinoside, cyanidin 3-0-glucoside, cyanidin 3-0-rutinoside), 
pelargonidins (cyanidin 3-0-(6-0-malonyl-beta-D-glucoside), cyanidin 3-0-arabinoside, cyanidin 3-0glucoside, cyanidin 3-0-rutinoside), peonidins (peonidin 3,5-0-diglucoside and peonidin 3-0-glucoside), proanthocyanidins (procyanidin B1, procyanidin B2, procyanidin B3, procyanidin $\mathrm{C} 1$ ), flavonols (quercetin 3-O-glucoside, fla_dihydrokaempferol) and petunidin 3-0-glucoside were identified as significantly upregulated during the red leaf period (RL) of $A$. mandshuricum (Fig. 2B). From Fig. S1, all the biological duplication groups were successfully grouped together, which indicated that the metabolic group data were highly reliable. In addition, it was obvious that the 9 samples were separated into two classes at the metabolite level, green class $(\mathrm{GL})$ and deep-colored (HRL and $\mathrm{RL}$ ) samples, and there were great differences in the patterns of metabolite accumulation between the two classes (Fig. S1).

To identify differential metabolites differentially accumulated metabolites (DAMs) between the different samples (GL vs HRL, GL vs HRL_RL, GL vs RL, HRL vs RL) in A. mandshuricum, the metabolites with VIP $\geq 1$ and fold change $\geq 2$ or $\leq 0.5$ were selected, and the heatmap and Venn diagram were used to analyze the differences and expression levels of different metabolites more intuitively and quickly. The results showed that there were significant differences in metabolites between the groups in the three leaf color changes, including 8 (8 up-regulated), 8 (8 up-regulated), 10 (10 up-regulated) and 7 (7 up-regulated) DAMs in GL vs HRL, GL vs HRL, GL vs HRL-RL, GL vs RL, and HRL vs RL, respectively (Table S2). It is worth mentioning that DAMs in the anthocyanin biosynthesis pathway of the four comparative groups were significantly increased (Fig. 2C-F), so we proposed that they were related to the leaf color changes of A. mandshuricum, especially cyanidin 3,5-0-diglucoside, cyanidin 3-0-(6-0-malonyl-beta-D-glucoside), cyanidin 3-0-arabinoside, cyanidin 3-0-glucoside, pelargonidin 3-0-glucoside, peonidin 3-0-glucoside, and petunidin 3-0-glucoside. In the Venn diagram (Fig. 2G), 5 anthocyanins were the common metabolites among green-colored and deep-colored samples, and all of them were upregulated and enriched significantly in the anthocyanin biosynthesis pathway (ko00942) (Table S3). Therefore, we speculated that those DAMs produced in the biosynthetic pathways of anthocyanins might be key metabolites during the leaf color change periods of $A$. mandshuricum.

Analysis of transcriptome data. Throughout the leaf color change cycle of $A$. mandshuricum, lllumina HiSeq 2000 was used to construct and sequence nine cDNA libraries based on the total RNAs of green (GL), half-red (HRL) and red (RL) leaf samples of $A$. mandshuricum. A total of $378,613,872$ raw reads and $368,399,930$ clean reads were obtained, and in the clean reads, the average Q20, Q30 and GC content was $97.63 \%, 93.42 \%$ and $43.89 \%$, respectively (Table S4). Then, gene function annotation was carried out, and we used BLAST software to compare unigenes to the KEGG, NR, Swiss-Prot, GO, KOG, Trembl and Pfam databases. A total of 201,642 unigenes were successfully annotated, of which 150946 unigenes $(74.86 \%)$ were annotated in at least one database above (Table S5 and Fig. 3B). Among the 20162 unigenes, 96,552 (47.88\%) unigenes were grouped into 25 KOG classifications; the most highly represented was group R (general function prediction only), and group C (energy production and conversion), group $\mathrm{J}$ (translation, ribosomal structure and biogenesis), group $\mathrm{O}$ (posttranslational modifi-cation, protein turnover, chaperones) and $\mathrm{T}$ (signal transduction mechanisms) also shared a high percentage of genes (Fig. 3C). A total of 116,336 unigenes were categorized into three $\mathrm{GO}$ categories (biological process, cellular component and molecular function) and further divided into 60 major functional groups (Fig. 3D). 
To reflect the expression level of transcripts, FPKM was used as an indicator to measure the level of gene expression. Principal component analysis (PCA) of the samples showed that the biological replications of GL samples and RL samples were obviously clustered together and significantly separated from each other, but the three HRL samples were dispersed (Fig. 3A). Similar to the metabolomic analysis, there was a significant separation of samples with different colors, indicating that changes in the accumulation of metabolites from green to red leaves were closely controlled by differences in gene expression.

Differentially expressed genes between different colored leaves. DESeq226 was used to analyze the differential expression between different sample groups, and the results showed that a total of 6988 (3106 down-regulated and 3882 up-regulated), 18,597 (11,442 down-regulated and 7155 up-regulated), and 11,474 (9433 down-regulated and 2041 up-regulated) DEGs were obtained in the GL vs HRL, GL vs $\mathrm{RL}$, and HRL vs RL groups, respectively (Table S6). The differentially expressed genes (DEGs) were identified, and the results are shown in Fig. 4A. From the Venn diagram, 217 genes were the common DEGs in all comparison groups, suggesting that those genes across the three compared groups might be associated with the leaf color change of $A$. mandshuricum (Fig. 4B). Next, we performed GO and KEGG functional annotation and pathway analysis in the three groups and found that the top enriched KEGG terms in the GL vs HRL group were ko01100 (metabolic pathways), ko01110 (biosynthesis of secondary metabolites), ko03010 (ribosome), ko04626 (plant-pathogen interaction) and ko04075 (plant hormone signal transduction) (Fig. 4D). Among them, the metabolic pathways and biosynthesis of secondary metabolites were also abundant in the GL vs RL and HRL vs RL groups, indicating that these DEGs might play a key role in leaf color change pathways (Table S7). GO functional enrichment analysis of DEGs between GL vs RL and GL vs HRL revealed that flavonoid metabolic process (GO: 0009812) and flavonoid biosynthetic process (GO:0009813) were significantly enriched (Fig. 4C).

To gain insight into the dynamism of gene expression pattern changes over the leaf color change periods, k-means cluster analysis was performed using the FPKM value of $G L, H R L$, and RL transcriptome data. Six clusters of co-expressed genes with different expression trends during leaves of different colors were separated by cluster analysis, implying that each cluster may have similar molecular functions (Fig. 5A). Class 1 consisted of 6863 genes, and the expression trend first increased slowly and then decreased rapidly from green to red leaves. By contrast, class 3 contained 3169 genes with an opposite trend compared with class 1 . Class 2 encompassed 3479 genes displaying an increase in expression levels from GL to HRL but a decrease from HRL to RL; this trend was similar to class 4 ( 1752 genes). A total of 6541 genes were identified in class 5 , displaying weak decreases from GL to RL. The most special gene was class 6, which had 2899 genes and exhibited a significant increasing trend for expression level, indicating that they were positively associated with the color change from green to red in $A$. mandshuricum. Several transcription factors (TFs) were encoded by these genes in class 6 , predominantly NAC, MYB-related, RWP-RK, GARP-G2-like, bHLH and $\mathrm{C} 2 \mathrm{H} 2$, which might play significant roles in the regulation of gene expression levels involved in leaf coloration of $A$. mandshuricum (Fig. 5B).

\section{Modulation of phenylpropanoid, flavonoid, and anthocyanidin biosynthesis pathway genes during leaf} color change. Anthocyanin components determine the color of $A$. mandshuricum leaves. However, the key 
genes involved in $A$. mandshuricum anthocyanin synthesis have not been reported. In this study, we constructed phenylpropanoid, flavonoid, and anthocyanidin biosynthesis pathways in A. mandshuricum. Ninety DEGs were searched based on enrichment analysis (Table S8) and predicted the molecular mechanisms leading to red leaf coloration (Fig. 6). From the expression levels, most key pathway genes exhibited significant changes. Indeed, encoding PAL (11 DEGs), C4H (8 DEGs), 4CL (10 DEGs), CHS (4 DEGs), CHI (9 DEGs), DFR (9 DEGs), F3H (6 DEGs), F3'5'H (1 DEG), ANS (2 DEGs) and BZ1 (2 DEGs) enzymes were more highly expressed in $\mathrm{HRL}$ and $\mathrm{RL}$ than in $\mathrm{GL}$, denoting that those $\mathrm{DEGs}$ were the key genes involved in the red pigmentation of $A$. mandshuricum leaves. Conversely, the expression level of one PAL gene (Cluster-20561.130819) was significantly down-regulated from the phenylalanine to cinnamic acid route when the leaves changed from green to red. Similarly, two 4CL genes (Cluster20561.65497 and Cluster-20561.89667), one CHS gene (Cluster-20561.139874), one CHI gene (Cluster20561.110302), four F3H genes (Cluster-20561.105759, Clus-ter-20561.110898, Cluster-20564.154341 and Cluster-20561.94199), one ANS gene (Cluster-20561.91533), two BZ1 genes (Cluster-20561.143.657 and Cluster-20561.81061) and one UGT75C1 gene (Cluster-20561.66602) were also significantly downregulated in their key pathways, implying that silencing these genes was essential for the leaf change to red. In the meantime, some notable genes were also discovered, two C4H genes (Cluster-20561.26608 and Cluster-20561.35599), one 4CL gene (Cluster-20561.36167) and one BZ1 gene (Cluster20561.99239) were activated in HRL stages, but with low expression in GL and RL. The discovery of these key differentially-expressed genes suggested that they could play a pivotal role in the process of $A$. mandshuricum leaf discoloration.

Comparing metabolite accumulation in anthocyanin synthesis pathways (ko00941) (Fig. 7), five cyanidins (up-regulated), two delphinidins (one up-regulated and one down-regulated), four malvidins (two up-regulated and two down-regulated), one pelnidin (up-regulated), four pelargonidins (up-regulated), and four peonidins (up-regulated) were determined. Thus, in summary, our results confirmed that the key genes identified in the biosynthesis pathways of flavonoids and anthocyanins could play a key role in $A$. mandshuricum leaves changing to red. Existing research has confirmed that MYB-related MYB, bHLH, bZIP and other transcription factors (TFs) are widely involved in the regulation of phenyl propane metabolic pathways as regulatory proteins 27,28 . Analysis of the expression levels of TFs such as bHLH, bZIP and MYB showed that some of them were down-regulated over the red periods, whereas others were significantly up-regulated, implying that these TFs positively regulate flavonoid and anthocyanidin biosynthesis pathway genes (Table S9). Overall, these TFs bHLH, bZIP, MYB and more coordinately regulate those key genes, leading to the accumulation of anthocyanin metabolites (cyanidin 3-0arabinoside, cyanidin 3,5-0-diglucoside, cyanidin 3-0-glucoside, cyanidin 3-0-rutinoside, cyanidin 3-0-(60-malonyl-beta-D-glucoside), delphinidin 3-0-glucoside, malvidin 3,5-diglucoside, malvidin 3-0-rutinoside, pelnidin 3,5-0-diglucoside, pelargonidin 3-0-(6-0-malonyl-beta-D-glucoside), pelargonidin 3-0-galactoside, pelargonidin 3-0-glucoside, pelargonidin 3-0-rutinoside, peonidin 3,5-0-diglucoside, peonidin 3-0galactoside, peonidin 3-0-glucoside, petunidin 3-0-glucoside), giving rise to the beautiful red coloration of A. mandshuricum leaves. 
Analysis of transcriptome factors. In the biosynthesis process of anthocyanins, it is coregulated by many transcription factors29,30. In this study, we listed anthocyanidin biosynthesis-related TFs in $A$.

mandshuricum, and a total of 341 (106 up-regulated and 235 down-regulated), 454 (103 up-regulated and 351 down-regulated) and 981 (346 up-regulated and 635 down-regulated) differentially expressed TFs were identified in GL vs HRL, HRL vs RL and GL vs RL, respectively (Table S10). Among them, most represented TFs were annotated as those encoding $\mathrm{C} 2 \mathrm{H} 2$, zn-clus, bZIP, MYB-related, NAC, bHLH, C3H, SNF2, TRAF, WRKY, C2C2-GATA and MYB in the GL vs RL group. The most abundant TFs in GL vs HRL (46 up-regulated and 78 down-regulated) were $\mathrm{C} 2 \mathrm{H} 2$, NAC, bHLH, MYB-related, $\mathrm{C} 3 \mathrm{H}, \mathrm{B} 3-\mathrm{ARF}$, WRKY and MYB TFs. In the HRL vs RL comparison (9 up-regulated and 151 down-regulated), C2H2, AP2/ERF-ERF, bHLH, bZIP, C2C2-GATA, C3H, HMG, MYB and MYB-related TFs were the most annotated. Previous studies have shown that transcription factors, such as MYB, bHLH and bZIP, have a deeper effect on anthocyanin biosynthesis31,32, and we also found them in many transcription factors in $A$. mandshuricum. For example, most of the MYB TFs (such as Cluster-20561.80755, Cluster-20561.81808, Cluster20561.110291, Cluster-20561.85332 and Cluster-20561.98223) were co-upregulated with most of the key genes in flavonoid biosynthesis during the discoloration of the leaves in A. mandshuricum, and the similar co-upregulated phenomenon was also found in bHLH (Cluster-20561.117348, Cluster20561.26518, Clus-ter-20561.46232, Cluster-20561.92650, Cluster-20561.107315, and more) and bZIP (Cluster-20561.89129, Clus-ter-20561.98504, Cluster-20561.102544, Cluster-20561.141195, Cluster20561.92791 and so on) TFs, indicating that those MYBs, bHLHs and bZIPs were important positive regulators in leaf color change periods of $A$. mandshuricum. In addition, it is worth noting that in the transcription factors of the three comparison groups, most bHLHs and bZIPs were downregulated when the leaves changed from green to red, indicating that some of the down-regulated genes mediated negative regulation in $A$. mandshuricum anthocyanin biosynthesis (Table S9).

Conjoint analysis between transcriptome and metabolome. To better understand the relationship between genes and metabolites, we mapped the DEGs and DAMs to the KEGG path map at the same time. From the results, many DEGs and DAMs were significantly enriched in the anthocyanin biosynthesis pathway. At the same time, we selected those results in which the Pearson correlation coefficient was greater than 0.8 . A total of $14310 \mathrm{DEGs}$ associated with 26 metabolites were found, and most of them jointly regulated the synthesis of one or more metabolites. They were significantly associated with Cya-3,5-0diglu (6696 genes), Cya-3-0-(6-0-malonyl)-glu (6807 genes), Cya-3-0-ara (6884 genes), Cya-3-0-glu (6568 genes), Cya-3-0-rut (6872 genes), Del-3-0-rut (3789 genes), fla_dihydrokaempferol (6622 genes), fla_Quercetin-glu (6010 genes), Mal-3-0-(6-0-malonyl)-glu (3199 genes), Pel-3,5-0-diglu (6899 genes), Pel3-0-(6-0-malonyl)-glu (6769 genes), Pel-3-0-glu (6821 genes), Peo-3,5-0-diglu (6755 genes), Peo-3-0-glu (6827 genes), Pet-3-0-glu (6536 genes) and others (Table S11).

From the results of Conjoint analysis between these DEGs and their correlated metabolites, we found two key structural genes ANS (Cluster-20561.86285) and BZ1 (Cluster-20561.99238) in anthocyanidin biosynthesis pathway were significantly up-regulated when the leaves change to red, indicating that they might enhance accumulation of the predominant metabolite cyanidin 3-0-glucoside which is their downstream metabolite, and contributed the red formation of A. mandshuricum leaves (Fig. 6). 
qRT-PCR Validation of DEGs in transcriptome data. To further verify the reliability and accuracy of the transcriptome data, we initially screened 12 DEGs (Cluster-20561.96219, Cluster-20561.85494, Cluster20561.85765, Cluster-20561.85766, Cluster-20561.102785, Cluster-20561.65565, Clus-ter-20561.86285, Cluster-20561.99238 and Cluster-20561.66602) which associated with phenylpropanoid, flavonoid, and anthocyanidin biosynthesis to detect expression levels in GL, HRL and RL with qRT-PCR. The relative expression trends of these candidate genes were similar to those of RNA-seq, indicating the high reliability and accuracy of transcriptome data (Fig. 8). In line with our expectations, the most pivotal genes ANS (Cluster-20561.86285) and BZ1 (Cluster-20561.99238) in anthocyanin biosynthesis of $A$. mandshuricum, were highly expressed when leaves change from green to red, suggesting that they are the candidate key genes underlying the leaf color formation in A. mandshuricum.

\section{Discussion}

A. mandshuricum is of vital ornamental and economic value because of its beautiful colored leaves. Previous studies have shown that the main substances in A. mandshuricum red leaf extract pigments are anthocyanins and flavonoids2. In our present study, using UPLC and a widely targeted metabolomics approach, we investigated the changes in metabolites in three different colored leaf samples of $A$. mandshuricum, aiming to provide a more comprehensive understanding of the accumulation dynamics of anthocyanins in the process of leaf color change. From the results (Table S1), a total of 26 anthocyanidins were simultaneously detected and quantified, mainly cyanidins, pelargonidins, peonidins, malvidins, delphinidins, petunidins, proanthocyanidins and flavonoids, which showed that they played an important role in the critical period of leaf color change. Furthermore, 17 metabolites, including cya-3-0glu, cya-3,5-0-diglu, pel-3-0-glu, pet-3-0-glu and others, were up-regulated when the leaves changed from green to red. Most of them have been reported in other plant coloration studies23,33,34, indicating that these compounds might be responsible for plants turning red. Especially the metabolite cyanidin 3-0glucoside was found that significantly correlated with the color formation, was the predominant metabolite in anthocyanin biosynthesis of $A$. mandshuricum, and accumulated in large quantities during the plant red period and was associated with numerous DEGs (6568 genes) (R2 > 0.8) (Table S1, Table S10). Therefore, this metabolite, cyanidin 3-0-glucoside, was considered an anthocyanin related to red pigmentation in $A$. mandshuricum. Interestingly, similar results of cyanidin 3-0-glucoside were also reported in some plants with red leaves or fruit35-37.

The biosynthesis of anthocyanin metabolites is tightly controlled by some structural genes (i.e., PAL, CHI, $D F R, A N S$ and more), which are also regulated by some transcription factors (i.e., MYB, bHLH, and WD40)38,39. In the whole biosynthesis of anthocyanin metabolites, early biosynthetic genes ( $\mathrm{CHS}, \mathrm{CHI}$, $F 3 H$, etc.) lead to the production of flavonoids, while late biosynthetic genes (i.e., $D F R, A N S$ ) lead to the production of anthocyanins 40 . We revealed that most of the structural genes that regulated the phenylpropanoid, flavonoid, and anthocyanidin biosynthetic pathways in the leaf's change to red were highly active (Fig. 6). The expression levels of most genes encoding $P A L, C 4 H, 4 C L, C H S, C H I, F 3 H, D F R$, $F 3^{\prime} 5^{\prime} H$, ANS and $B Z 1$ were higher in RL than in GL, resulting in higher anthocyanin accumulation during the red leaf stage. $F 3^{\prime} H$ and $F 3^{\prime} 5^{\prime} H$ enzymes are important for anthocyanin accumulation, which could 
determine the B-ring hydroxylation pattern of anthocyanins41, and ANS and DFR genes have been proven to catalyze the conversion of colorless leucoanthocyanidins into available colored anthocyanidins 42 . In this study, we found that six F3H genes (Cluster-20561.126021, Cluster-20561.93983, Cluster20561.93984, Cluster-20561.98637, Cluster-20561.98639, Cluster-20561.98643), one F3'5'H gene (Cluster-20561.65565), nine DFR genes (Cluster-20561.102785, Cluster-20561.92388, Cluster20561.92390, Cluster-20561.92391, Cluster-20561.92394, Cluster-20561.92503, Cluster-20561.97639, Cluster-20561.97640, Cluster-20561.97641) and two ANS genes (Cluster-20561.86283 and Cluster20561.86285) were significantly up-regulated in RL, suggesting that they might enhance the red color accumulation, forming leucoanthocyanidin and transferring into anthocyanidins in A. mandshuricum. Previous studies have shown that the ANS gene is abundantly expressed in cell cultures of red cell lines and seedlings in the rare medicinal plant Saussurea medusa43, and the high expression of ANS and DFR also promotes anthocyanin biosynthesis in red pear fruit skin44; similar findings have been validated in Litchi chinensis Sonn45 and Malus hupehensis38. In addition, the anthocyanidin 3-0-glucosyltransferase (BZ1, EC: 2.4.1.115) is the last key enzyme in the anthocyanin biosynthetic pathway and can catalyze the transformation of unstable anthocyanidin into anthocyanin, including by catalyzing the transfer of glucose from UDP-glucose to anthocyanidins including delphinidin, cyanidin, etc.46-48. As the leaves changed from green to red, two $B Z 1$ genes were down-regulated, two genes were up-regulated, and one gene was up-regulated only in half-red leaves. Notably, the $B Z 1$ gene Cluster-20561.99238 (Table S8) was highly expressed, and its downstream metabolites (cyanidin 3-0-glucoside, pelargonidin 3-0-glucoside, and delphinidin 3-0-glucoside) were correspondingly significantly upregulated in the RL period (Fig. 6). Therefore, we suggested that $B Z 1$ was the key gene for regulating anthocyanin synthesis and leaf color stabilization. Interestingly, we found that the UGT75C1 gene (anthocyanidin 3-0-glucoside 5-0glucosyltransferase, EC:2.4.1.298) was significantly down-regulated in the anthocyanin synthesis process, leading to the formation of more derivatives of anthocyanins.

Some TFs, such as MYB, bHLH, and WD40, are key regulatory factors for structural genes in flavonoid biosynthesis pathways49-50. The MYB transcription factor family is very large and versatile in plants, and these TFs can regulate the biosynthesis of phenylpropanoids51. Therefore, many MYB genes were identified, which were considered potential key regulators in the anthocyanin biosynthesis pathway52. MYB, bHLH, and WD40 can be combined into the transcription factor complex MBW (MYB-bHLH-WD40) to regulate the biosynthesis of anthocyanins53; they have been shown to regulate the synthesis of anthocyanins positively or negatively in plants including banana 54 , rice 55 , persimmon 56 , etc. In the present study, a total of 341 (106 up-regulated and 235 down-regulated), 454 (103 up-regulated and 351 down-regulated) and 981 (346 up-regulated and 635 down-regulated) differentially expressed TFs were identified in GL vs HRL, HRL vs RL and GL vs RL, respectively (Table S9). Among them, we identified several candidate MYB (Cluster-20561.80755, Cluster-20561.81808, Cluster-20561.96473, and Cluster20561.98223) and bHLH (Cluster-20561.92650) TFs that were highly up-regulated with most of the key genes in flavonoid biosynthesis during the discoloration of the leaves in A. mandshuricum and may positively regulate the key structural genes in anthocyanin synthesis in A. mandshuricum (Table S8). In addition, some TFs such as NAC, RWP-RK. WRKY, C2H2, GARP-G2-like, MYB-related, HD-Zip, and bZIP 
were discovered during the leaf color change periods and displayed different expression levels, indicating that they may co-regulate the expression level of structural genes involved in the synthesis of flavonoids, similar to the results of recent studies 57,58 .

\section{Materials And Methods}

Plant materials. Fresh plant leaves of $A$. mandshuricum were harvested during three consecutive leaf color change periods, i.e., green leaf (GL), half red leaf (HRL) (the transition period from green leaves to red leaves), and red leaf (RL) (Fig. 1). The samples were frozen in liquid nitrogen and stored at $-80^{\circ} \mathrm{C}$ until further use. Each color leaf contains three biological repeats. For each repeat, normal growth and no pests or diseased leaves were considered for one mixed sample33, and the total sample weight was more than $3 \mathrm{~g}$. These samples were used for subsequent transcriptome and metabolome studies.

Fresh samples (not wild plant) were collected at the Northeast Forestry University (North: 45.723999; East: 126.635474) in Heilongjiang Province, China, which had been approved by the Chinese government and implemented in compliance with the laws of the People's Republic of China.

Sample preparation and metabolite extraction. For metabolite extraction, five steps needed to be completed. First, the samples were subjected to vacuum freeze-drying. Second, a mixer mill (MM 400, Retsch) was used to crush the freeze-dried samples with a zirconia bead for $1.5 \mathrm{~min}$ at $30 \mathrm{~Hz}$. Then, 50 $\mathrm{mg}$ powder was weighed and extracted with $0.5 \mathrm{ml}$ methanol/water/hydrochloric acid (799:200:1, $\mathrm{V} / \mathrm{V} / \mathrm{V})$. The next was step repeated twice: the extractives were vortexed for $10 \mathrm{~min}$, ultrasonicated for 10 min, and centrifuged at $12000 \times \mathrm{g}$ under $4^{\circ} \mathrm{C}$ for $3 \mathrm{~min}$. Finally, the supernatants were collected and filtered (PTFE, $0.22 \mu \mathrm{m}$; Anpel) before LC-MS/MS analysis. Professional analytical processes of LCMS/MS were performed by MetWare (http://www.metware.cn/), using the same methods described in previous articles59.

Metabolite analysis. Metabolite data analysis was conducted with the R Programming language (https://www.r-project.org/), and hierarchical cluster analysis (HCA) and orthogonal partial least-squares discriminant analysis (OPLS-DA) were performed to identify the ac-cumulation pattern in metabolites from nine leaf samples of three colors. Furthermore, the metabolic composition differences between different samples were observed by the PLS-DA model with a variable importance in projection (VIP) value (VIP $\geq 1)$ and fold change $\geq 2$ or $\leq 0.5$.

RNA extraction, quantification and sequencing. RNA extraction, quantification and transcriptome sequencing were performed as fully described in Lu's study60. For transcriptome sequencing, nine libraries representing the three leaf samples and three replicates were constructed. All libraries were sequenced on an Illumina HiSeq platform, and raw data with 150 bp paired-end reads were obtained for subsequent analysis.

Transcriptome data analysis. The raw data (or raw reads) were filtered with FASTP software (version 0.12 ) to filter out adapters and low-quality sequences and obtain high-quality clean sequences. Then, we 
used Trinity software to perform De novo assembly. To obtain the annotation information, the unigenes were compared with public databases, including KEGG (Kyoto Encyclopedia of Genes and Genomes), GO (Gene Ontology), KOG (euKaryotic Ortholog Groups), NR (Non-Redundant Protein Sequence Database), Swiss-Prot (a manually annotated and reviewed protein sequence database), Pfam (protein family) and Trembl. FPKM (fragments per kilobase of transcript per million fragments mapped) was used as an indicator to measure the level of transcript or gene expression. Differential expression analysis between different comparisons of colored samples was performed using the DESeq2 R package (1.20.0)61. When the difference analysis was completed, the Benjamini-Hochberg method was used to carry out multiple hypothesis test corrections for the $\mathrm{P}$ value to obtain the false discovery rate (FDR). The screening conditions of unigenes were $\mid \log 2$ fold change $(F C) \mid \geq 1$ and FDR $<0.05$. KEGG and GO enrichment analyses of differentially expressed genes were further implemented employing the KOBAS 2.0 software62 and G0seq R packages63, respectively.

Gene validation by real-time quantitative PCR (qRT-PCR). To further verify the reliability and accuracy of the transcriptome data, we initially screened 12 DEGs associated with phenylpropanoid, flavonoid, and anthocyanidin biosynthesis to detect expression levels in GL, HRL and RL with qRT-PCR. The Tiangen total RNA extraction kit (Tiangen, Beijing, China) and 209 PrimeScript RT reagent Kit with gDNA Eraser (TaKaRa, Kyoto, Japan) were used for RNA extraction and cDNA synthesis, respectively. Primer 3 software (https://primer3.ut.ee/) was used to design specific primers for qRT-PCR analysis. qRT-PCR was carried out with the TaKaRa SYBR Green Mix kit (TaKaRa, Beijing, China) using an ABI 7500 Fast RealTime Detection System. The PCR amplification experiment was performed with a total volume of $20 \mu \mathrm{l}$ $(10 \mu \mathrm{l}$ 2xSYBR Primix ExTaq, $0.4 \mu \mathrm{l}$ Rox-Rfarfebce, $6 \mu \mathrm{lddH} 20,0.8 \mu$ l downstream primer, $0.8 \mu$ upstream primer, and $2 \mu \mathrm{l} \mathrm{cDNA}$ template under a standard program $\left(95^{\circ} \mathrm{C}\right.$ for $30 \mathrm{~s}, 40$ cycles at $95^{\circ} \mathrm{C}$ for $5 \mathrm{~s}, 60^{\circ} \mathrm{C}$ for $35 \mathrm{~s}$, and $95^{\circ} \mathrm{C}$ for $15 \mathrm{~s}$, and $60^{\circ} \mathrm{C}$ for $1 \mathrm{~min}$, followed by $95^{\circ} \mathrm{C}$ for $15 \mathrm{~s}$ ). The relative expression analysis of quantitative data was performed using the $2-\Delta \Delta C T$ method with reference gene 18S-RNA as control.

\section{Conclusion}

In this study, the molecular mechanisms of anthocyanin biosynthesis in A. mandshuricum during different leaf color change periods were revealed based on metabolomics and transcriptomics studies. We analyzed the accumulation pattern of anthocyanins in leaf color change periods in three different leaf color states and found that cyanidin 3-0-glucoside was the predominant metabolite in the biosynthesis of the anthocyanin synthesis pathway, which accumulated in large quantities during the red plant period. Furthermore, we analyzed the differentially expressed genes associated with anthocyanin biosynthesis in the leaves at different times. A variety of regulatory models of structural genes ( $P A L, A N S, B Z 1$, etc.) and some potential transcription factors (MYB, bHLH, C2H2, NAC, bZIP, etc.) involved in biosynthesis of flavonoid pathways and anthocyanin biosynthesis pathway were found. The regulation of these key structural genes or their transcription factors might help elucidate the mechanism of leaf color change in A. mandshuricum. Our studies could provide novel insight into the molecular regulation of anthocyanin biosynthesis and accumulation associated with leaf color change in A. mandshuricum. 


\section{Declarations}

\section{Acknowledgements}

This work was supported by the Heilongjiang Province Applied Technology Research and Development Plan Project (No. GA20B402), Fundamental Research Funds for the Central Universities (Northeast Forestry University) (No. 2572020DR01) and Heilongjiang Touyan Innovation Team Program (Tree Genetics and Breeding Innovation Team).

\section{Author contributions statement}

X.Z. (Xiyang Zhao), S.Z. (Shikai Zhang) and W.Z. (Wang Zhan) conceived the experiment(s) and wrote the manuscript, A.S. (Anran Sun) and Y.X. (Ying Xie), Z.H. (Zhiming Han), X.Q. (Xibin Qu) and Y.J. (Jiayi Wang) conducted the experiment(s) and analysed the results. J.Z. (Jinbao Zhang) and M.T. (Mingshun Tian) consulted literatures and revised the grammar, L.Z. (Laifu Zhang) and X.P. (Xuhong Pang) made the final revision to the article. All authors reviewed the manuscript and agreed to the published version of the manuscript.

\section{Additional information Competing interests}

Data Availability Statement. Transcriptome sequencing data have not yet been uploading at the time of submission, we will be provided it during review. It will be provided prior to publication.

\section{Competing interests}

The authors declare no conflict of interest.

\section{References}

1. Zhu, Y. Y. Research on relationships between functional traits and compensation mechanisms of androdioecious Acer mandshuricum, master thesis, Beijing Forestry University(2016).

2. Liu, X. L. Research on red pigment in leaves and leaves color retention of Acer mandshuricum, master thesis, Northeast Forestry University(2014).

3. Xie, Z., Yi, X. Y. \& Xia, F. C. The resource situation and development utilization of Acer plants in northeast area. Journal of Jilin Forestry Science and Technology, 46, 26-31 https://doi.org/10.16115/j.cnki.issn.1005-7129.2017.01.007 (2017).

4. Reay, P. F., Fletcher, R. H. \& Thomas, V. J. Chlorophylls, carotenoids and anthocyanin concentrations in the skin of 'Gala' apples during maturation and the influence of foliar applications of nitrogen and magnesium. Journal of the Science of Food \& Agriculture, 76, 63-71 https://doi.org/10.1002/(SICI)1097-0010(199801)76 (2015). 
5. Englert, M., Hammann, S. \& Vetter, W. Isolation of $\beta$-carotene, a-carotene and lutein from carrots by countercurrent chromatography with the solvent system modifier benzotrifluoride. Journal of Chromatography A, 1388, 119-125 https://doi.org/10.1016/j.chroma.2015.02.020 (2015).

6. Grotewold, E. The genetics and biochemistry of floral pigments. Annual Review of Plant Biology, 57, 761-780 https://doi.org/10.1146/annurev.arplant.57.032905.105248 (2006).

7. Zhao, M. H., Li, X., Zhang, X. X., Zhang, H. \& Zhao, X. Y. Mutation Mechanism of Leaf Color in Plants. A Review. Forests, 11, https://doi.org/10.3390/f11080851 (2020).

8. Turturică, M., Oancea, A. M., Râpeanu, G., Bahrim, G. \& Anthocyanins Naturally occuring fruit pigments with functional properties. Annals of the University Dunarea de Jos of Galati. Fascicle VI: Food Technology(2015).

9. Tanaka, S. \& Ohmiya Biosynthesis of plant pigments: anthocyanins, betalains and carotenoids. PLANT J 2008,54(4), 733-749, DOI: 10.1111/j.1365-313X.2008.03447.x (2008).

10. He, Q. et al. Natural Variation in Petal Color in Lycoris longituba Revealed by Anthocyanin Components., 6, e22098 https://doi.org/10.1371/journal.pone.0022098 (2011).

11. Martins, T. R., Berg, J. J., Blinka, S., Rausher, M. \& Baum, D. A. Precise spatio-temporal regulation of the anthocyanin biosynthetic pathway leads to petal spot formation in Clarkia gracilis (Onagraceae). New Phytol, 197, https://doi.org/10.1111/nph.12062 (2013).

12. Sheng, T., Keurentjes, J., Bentsink, L. \& Smeekens, K. S. Sucrose-specific induction of anthocyanin biosynthesis in Arabidopsis requires the MYB75/PAP1 gene. Plant Physiol, 139, 1840-1852 https://doi.org/10.1104/pp.105.066688 (2005).

13. Wei, S. et al. Molecular and Biochemical Analysis of Chalcone Synthase from Freesia hybrid in Flavonoid Biosynthetic Pathway., 10, e0119054 https://doi.org/10.1371/journal.pone.0119054 (2015).

14. Wei, H. et al. Comparative Transcriptome Analysis of Genes Involved in Anthocyanin Biosynthesis in the Red and Yellow Fruits of Sweet Cherry (Prunus avium L.)., 10, e0121164 https://doi.org/10.1371/journal.pone.0121164 (2015).

15. Watts, K. T., Lee, P. C. \& Schmidt-Dannert, C. Exploring Recombinant Flavonoid Biosynthesis in Metabolically Engineered Escherichia coli (p500-507)., 5, 500-507 https://doi.org/10.1002/cbic.200300783 (2010).

16. Liang, J. \& He, J. Protective role of anthocyanins in plants under low nitrogen stress. Biochem Biophys Res Commun, 946-953 https://doi.org/10.1016/j.bbrc.2018.03.087 (2018).

17. Huang, Y., Zhang, J., Fan, Y., Liu, Y. \& Yang, M. Effects of Shading Treatmentson Leaf Color and Related Physiological Indexes of Ulmus pumila 'Jinye' and Koelreuteria paniculata 'Xinye'. Scientia Silvae Sinicae, 55, 171-180 (2019).

18. Kim, J., Woo, H. R. \& Hong, G. N. TowardSystems Understanding of Leaf Senescence: An Integrated Multi-Omics Perspective on Leaf Senescence Research. Mol. Plant, 9, 813-825 https://doi.org/10.1016/j.molp.2016.04.017 (2016). 
19. Zhang, C., Wang, M., Gao, X., Zhou, F. \& Liu, Z. Multi-omics research in albino tea plants: Past, present, and future. Sci. Hort, 261, 108943 https://doi.org/10.1016/j.scienta.2019.108943 (2020).

20. Liu, X., Zhang, J., Luo, Y. \& Rao, G. Metabolome and Transcriptome Analyses Reveal Tissue-Specific Variations in Gene Expression and Metabolites of Olive. Journal of Plant Biology, 63, 73-82 https://doi.org/10.1007/s12374-020-09231-z (2020).

21. Sun, J. T. Sequencing and Metabolome Analysis Reveal Genes Involved in Pigmentation of GreenColored Cotton Fibers. International Journal of Molecular Sciences, 20, 4838 https://doi.org/10.3390/ijms20194838 (2019).

22. Fang-Fang, F. U., Peng, Y. S., Wang, G. B., El-Kassaby, Y. A. \& Cao, F. L. Integrative analysis of the metabolome and transcriptome reveals seed germination mechanism in Punica granatum L. Journal of Integrative Agriculture, 20, 132-146 https://doi.org/10.1016/S2095-3119(20)63399-8 (2021).

23. Wan, L., Lei, Y., Yan, L., Liu, Y. \& Liao, B. Transcriptome and metabolome reveal redirection of flavonoids in a white testa peanut mutant. BMC Plant Biology, 20, https://doi.org/10.1186/s12870020-02383-7 (2020).

24. Qiu, W., Su, W., Cai, Z., Dong, L. \& Wu, Z. Combined Analysis of Transcriptome and Metabolome Reveals the Potential Mechanism of Coloration and Fruit Quality in Yellow and Purple Passiflora edulis Sims. Journal of agricultural and food chemistry, 68, 12096-12106 https://doi.org/10.1021/acs.jafc.0c03619 (2020).

25. Wei, Y., Zhang, Y., Meng, J., Wang, Y. \& Zhong, C. Transcriptome and metabolome profiling in naturally infested Casuarina equisetifolia clones by Ralstonia solanacearum., https://doi.org/10.1016/j.ygeno.2021.03.022 (2021).

26. Zhang, Q., Wang, L., Liu, Z., Zhao, Z. \& Liu, M. Transcriptome and metabolome profiling unveil the mechanisms of Ziziphus jujuba Mill. peel coloration. Food Chem, 312, 125903 https://doi.org/10.1016/j.foodchem.2019.125903 (2019).

27. Qin, G., Liu, C., Li, J., Qi, Y. \& Xu, Y. Diversity of metabolite accumulation patterns in inner and outer seed coats of pomegranate: exploring their relationship with genetic mechanisms of seed coat development. Horticulture Research, 7, 10 https://doi.org/10.1038/s41438-019-0233-4 (2020).

28. Lu, D. et al. Transcriptome analysis of chrysanthemum in responses to white rust. Sci. Hort, 233, 421-430 https://doi.org/10.1016/j.scienta.2018.01.016 (2018).

29. Love, M. I., Huber, W. \& Anders, S. Moderated estimation of fold change and dispersion for RNA-seq data with DESeq2. Genome Biol, 15, 550 https://doi.org/10.1186/s13059-014-0550-8 (2014).

30. Chen, X. et al. KOBAS 2.0: a web server for annotation and identification of enriched pathways and diseases. Nucleic Acids Res, 39, 316-322 https://doi.org/10.1093/nar/gkr483 (2011).

31. Young, M., Wakefield, M. J., Smyth, G. K. \& Oshlack, A. Gene ontology analysis for RNA-seq: accounting for selection bias. Genome Biol, 11, R14-R14 https://doi.org/10.1186/gb-2010-11-2-r14 (2010).

32. Varet, H., Brillet-Guéguen, L., Coppée, J., Dillies, M. A. \& SARTools: A DESeq2- and EdgeR-Based R Pipeline for Comprehensive Differential Analysis of RNA-Seq Data., 11, 
https://doi.org/10.1371/journal.pone.0157022 (2016).

33. Yamagishi, M. Involvement of a LhMYB18 transcription factor in large anthocyanin spot formation on the flower tepals of the Asiatic hybrid lily (Lilium spp.) cultivar "Grand Cru". Mol. Breeding, 38, https://doi.org/10.1007/s11032-018-0806-1 (2018).

34. Filiz, E. \& Kurt, F. Expression and Co-expression Analyses of WRKY, MYB, bHLH and bZIP Transcription Factor Genes in Potato (Solanum tuberosum) Under Abiotic Stress Conditions: RNAseq Data Analysis. Potato Res, 1-21 https://doi.org/10.1007/s11540-021-09502-3 (2021).

35. Marie, P. \& Burow Meike \& Regulation of MYB and bHLH Transcription Factors: A Glance at the Protein Level. Molecular Plant, DOI: 10.1016/j.molp.2014.11.022 (2015).

36. Zongyan, L. I. \& Mingyang, L. I. Advance in transcriptional factors regulating flavonoid biosynthesis. Journal of Nanjing Forestry University(Natural Sciences Edition), DOI: 10.1631/jzus.B1000171 (2011).

37. Duan, S. et al. Functional characterization of a heterologously expressed Brassica napus WRKY41-1 transcription factor in regulating anthocyanin biosynthesis in Arabidopsis thaliana. Plant Sci, 268, 47-53 https://doi.org/10.1016/j.plantsci.2017.12.010 (2018).

38. Li, X., Xiang, F., Han, W., Qie, B. \& Xu, L. The MIR-Domain of PbbHLH2 Is Involved in Regulation of the Anthocyanin Biosynthetic Pathway in "Red Zaosu" (PyrusBretschneideri Rehd.) Pear Fruit. International Journal of Molecular Sciences, 22, 3026 https://doi.org/10.3390/ijms22063026 (2021).

39. Chen, Z. et al. Transcriptome analysis based on a combination of sequencing platforms provides insights into leaf pigmentation in Acer rubrum. BMC Plant Biol, 19, https://doi.org/10.1186/s12870019-1850-7 (2019).

40. cker, C. B., Klaering, H. P., Kroh, L. W. \& Krumbein, A. Cool-cultivated red leaf lettuce accumulates cyanidin-3-0-(6 "-0-malonyl)-glucoside and caffeoylmalic acid. Food Chem, 146, 404-411 https://doi.org/10.1016/j.foodchem.2013.09.061 (2014).

41. Gao, Y. F. et al. De novo transcriptome sequencing and anthocyanin metabolite analysis reveals leaf color of Acer pseudosieboldianum in autumn. BMC Genomics, 22 (1), https://doi.org/10.1186/s12864-021-07715-x (2021).

42. Wang, X., Bai, J., Wang, W. \& Zhang, G. Leaf metabolites profiling between red and green phenotypes of Suaeda salsa by widely targeted metabolomics. Funct. Plant Biol, 46, https://doi.org/10.1071/FP18182 (2019).

43. Han, M., Yang, C., Zhou, J., Zhu, J. \& Li, H. Analysis of flavonoids and anthocyanin biosynthesisrelated genes expression reveals the mechanism of petal color fading of Malus hupehensis (Rosaceae). Brazilian Journal of Botany, https://doi.org/10.1007/s40415-020-00590-y (2020).

44. Li, T., Zou, H. \& Tang The Effects of Ultraviolet A/B Treatments on Anthocyanin Accumulation and Gene Expression in Dark-Purple Tea Cultivar 'Ziyan' (Camellia sinensis)., 25, 354 https://doi.org/10.3390/molecules25020354 (2020).

45. Zhang, Y. Z., Xu, S. Z., Cheng, Y. W., Ya, H. Y. \& Han, J. M. Transcriptome analysis and anthocyaninrelated genes in red leaf lettuce. Genetics \& Molecular Research Gmr, 15, 
https://doi.org/10.4238/gmr.15017023 (2016).

46. Huang et al. Flower Colour Modification of Chrysanthemum by Suppression of F3 ' H and Overexpression of the Exogenous Senecio cruentus F3 ' 5 ' H Gene. Plos One 2013,8(11), DOI: 10.1371/journal.pone.0074395 (2013).

47. Han et al. Introduction of apple ANR genes into tobacco inhibits expression of both $\mathrm{CHI}$ and DFR genes in flowers, leading to loss of anthocyanin. Journal of Experimental Botany, 63 (7), 2437-2447 https://doi.org/10.1093/jxb/err415 (2012).

48. Cheng, L. et al. Characterization of Anthocyanidin Synthase (ANS) Gene and anthocyanidin in rare medicinal plant-Saussurea medusa. Plant Cell Tissue \& Organ Culture, 89, 63-73 https://doi.org/10.1007/s11240-007-9211-x (2007).

49. Li, X., Wu, T., Liu, H., Zhai, R. \& Xu, L. REVEILLE Transcription Factors Contribute to the Nighttime Accumulation of Anthocyanins in 'Red Zaosu' (Pyrus bretschneideri Rehd.) Pear Fruit Skin. International Journal of Molecular Sciences, 21, 1634 https://doi.org/10.3390/ijms21051634 (2020).

50. Wei, Y. Z. et al. Differential Expression of Anthocyanin Biosynthetic Genes in Relation to Anthocyanin Accumulation in the Pericarp of Litchi Chinensis Sonn. PLoS One, 6, e19455 https://doi.org/10.1371/journal.pone.0019455 (2011).

51. Hiromoto, T. et al. Structural basis for acceptor-substrate recognition of UDP-glucose: anthocyanidin 3-0-glucosyltransferase from Clitoria ternatea. Protein Sci, 24, 395-407 https://doi.org/10.1002/pro.2630 (2015).

52. Du, L. J., Chen, K. L. \& Liu, Y. L. Cloning and expression analysis of anthocyanidin 3-Oglucosyltransferase gene in Grape hyacinth. Pratacultural Science, 34 (11), 2235-2244 (2017).

53. Hongxia et al. UDP-glucose:anthocyanidin 3-0-glucoside-2"-0-glucosyltransferase catalyzes glycosyl extension of anthocyanins in purple Ipomoea batatas. Journal of Experimental Botany, https://doi.org/10.1093/jxb/ery305 (2018).

54. Cui, X., Deng, J., Huang, C., Tang, X. \& Zhang, Z. Transcriptomic Analysis of the Anthocyanin Biosynthetic Pathway Reveals the Molecular Mechanism Associated with Purple Color Formation in Dendrobium Nestor. Life, 11, 113 https://doi.org/10.3390/life11020113 (2021).

55. Yao, Y., Sun, L., Wu, W. J., Wang, S. \& Wu, Q. Genome-Wide Investigation of Major Enzyme-Encoding Genes in the Flavonoid Metabolic Pathway in Tartary Buckwheat (Fagopyrum tataricum). Journal of Molecular Evolution, 1-18 https://doi.org/10.1007/s00239-021-10004-6 (2021).

56. Arce-Rodríguez, M., Martínez, O. \& Ochoa-Alejo, N. Genome-Wide Identification and Analysis of the MYB Transcription Factor Gene Family in Chili Pepper (Capsicum spp.). International Journal of Molecular Sciences, 22, 2229 https://doi.org/10.3390/ijms22052229 (2021).

57. Zhu, Y. \& Bao, Y. Genome-Wide Mining of MYB Transcription Factors in the Anthocyanin Biosynthesis Pathway of Gossypium Hirsutum. Biochem. Genet, 59, 678-696 https://doi.org/10.1007/s10528021-10027-0 (2021).

58. Liu, Y. et al. Comparative Transcriptome Analysis of White and Purple Potato to Identify Genes Involved in Anthocyanin Biosynthesis., 10, e0129148 https://doi.org/10.1371/journal.pone.0129148 
(2015).

59. Deng, G. M., Zhang, S., Yang, Q. S., Gao, H. J. \& Hu, C. H. MaMYB4, an R2R3-MYB Repressor Transcription Factor, Negatively Regulates the Biosynthesis of Anthocyanin in Banana. Frontiers in Plant Science, 11, 600704 https://doi.org/10.3389/fpls.2020.600704 (2021).

60. Qiao, W. H. et al. A functional chromogen gene $\mathrm{C}$ from wild rice is involved in a different anthocyanin biosynthesis pathway in indica and japonica. Theoretical and Applied Genetics, 134, 1531-1543 https://doi.org/10.1007/s00122-021-03787-1 (2021).

61. Gil-Muoz, F., Sánchez-Navarro, J., Besada, C., Salvador, A. \& Ríos, G. MBW complexes impinge on anthocyanidin reductase gene regulation for proanthocyanidin biosynthesis in persimmon fruit. Sci. Rep, 10, https://doi.org/10.1038/s41598-020-60635-w (2020).

62. Ahmad, S. et al. Transcriptional Cascade in the Regulation of Flowering in the Bamboo Orchid Arundina graminifolia, Biomolecules 11(6), DOI: 10.3390/biom11060771 (2021).

63. Premathilake, A. T., Ni, J., Shen, J., Bai, S. \& Teng, Y. Transcriptome analysis provides new insights into the transcriptional regulation of methyl jasmonate-induced flavonoid biosynthesis in pear calli. BMC Plant Biology, 20, https://doi.org/10.1186/s12870-020-02606-x (2020).

\section{Figures}
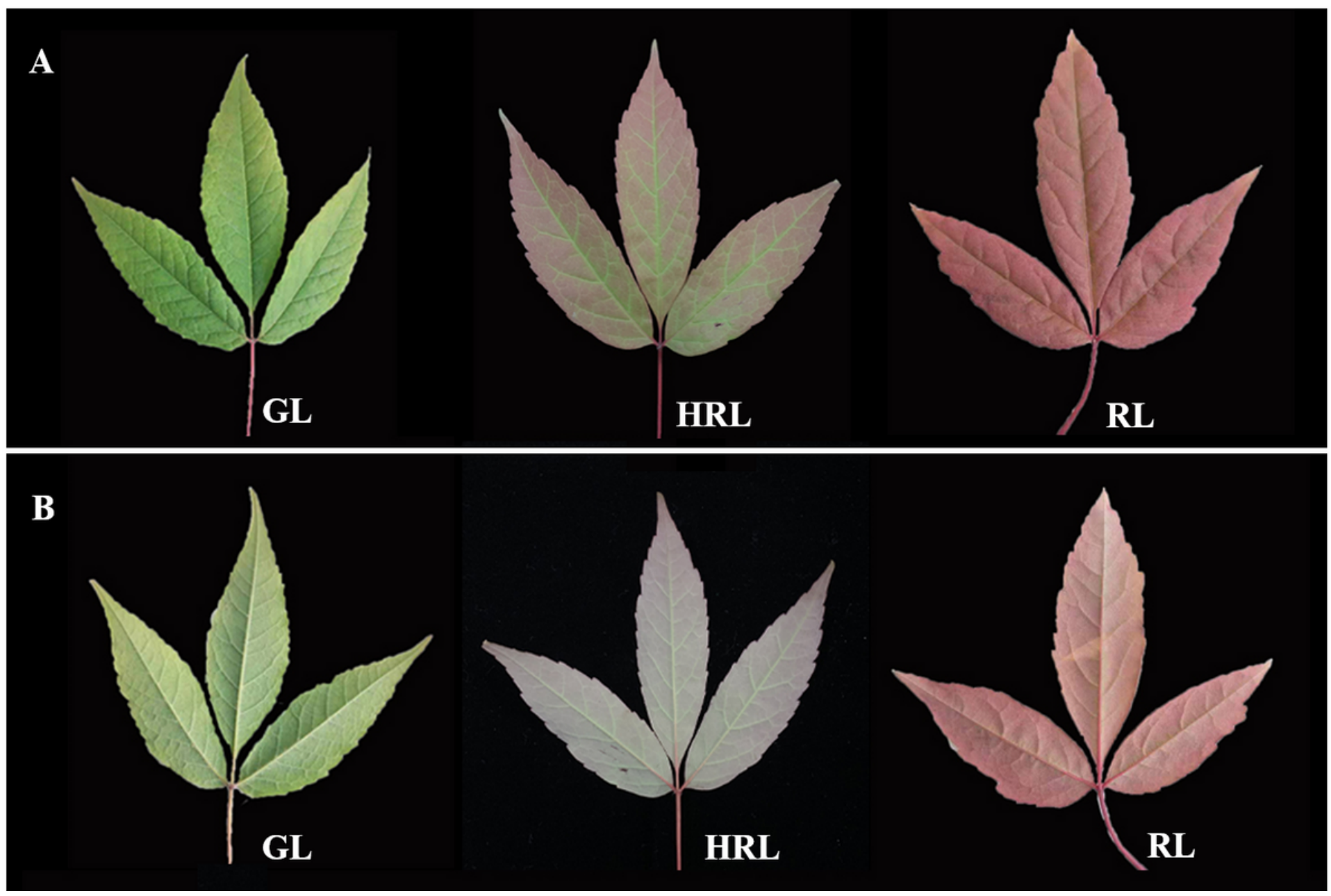
Figure 1

Phenotypes of $A$. mandshuricum. Green (GL), half red leaf (HRL), red (RL) leaves on front $(A)$ and back (B).
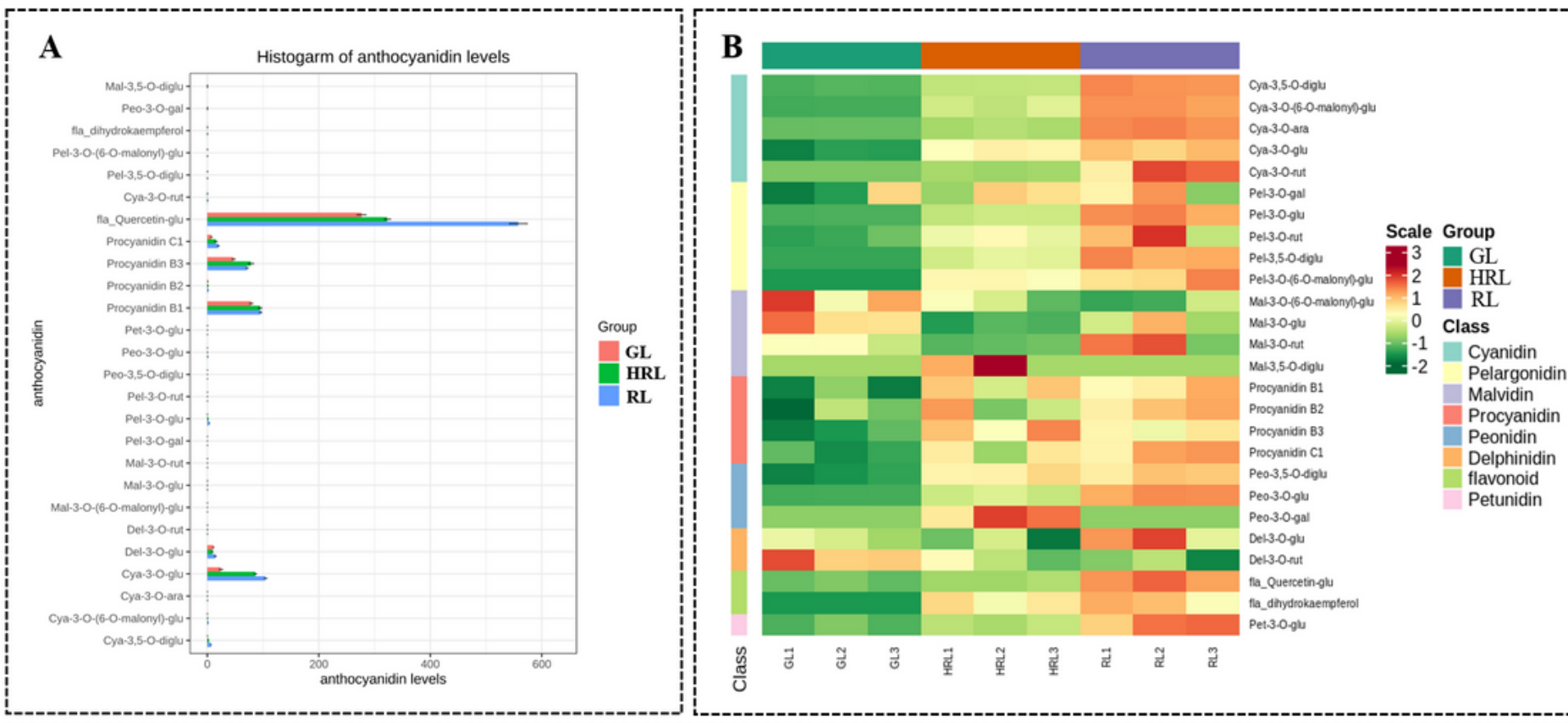

C

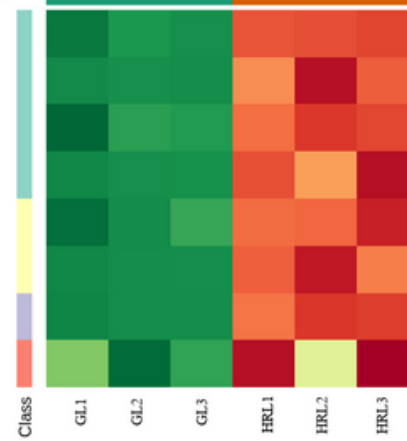

E

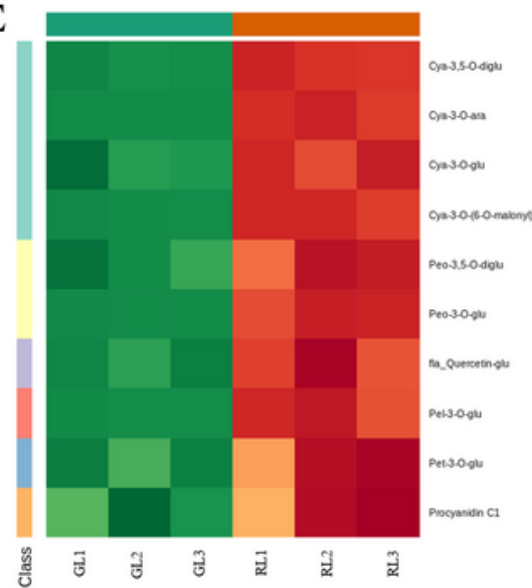

D

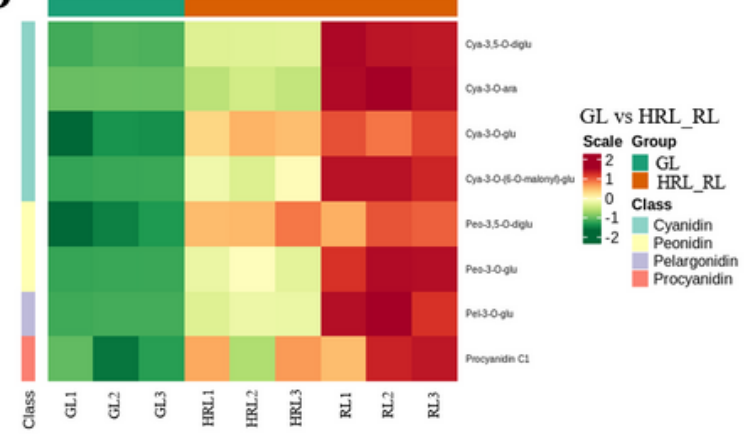

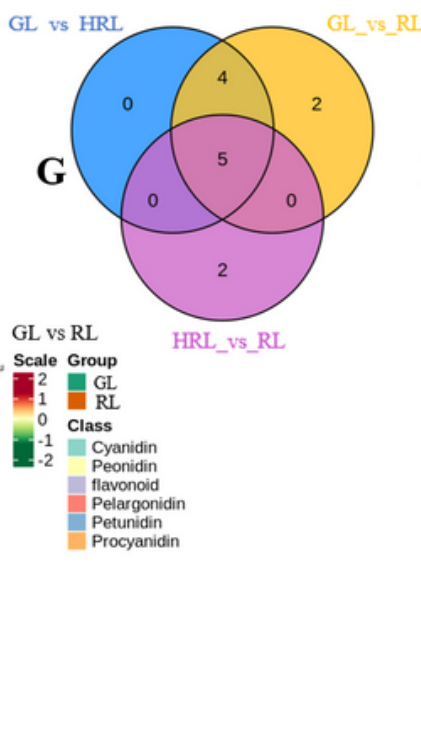

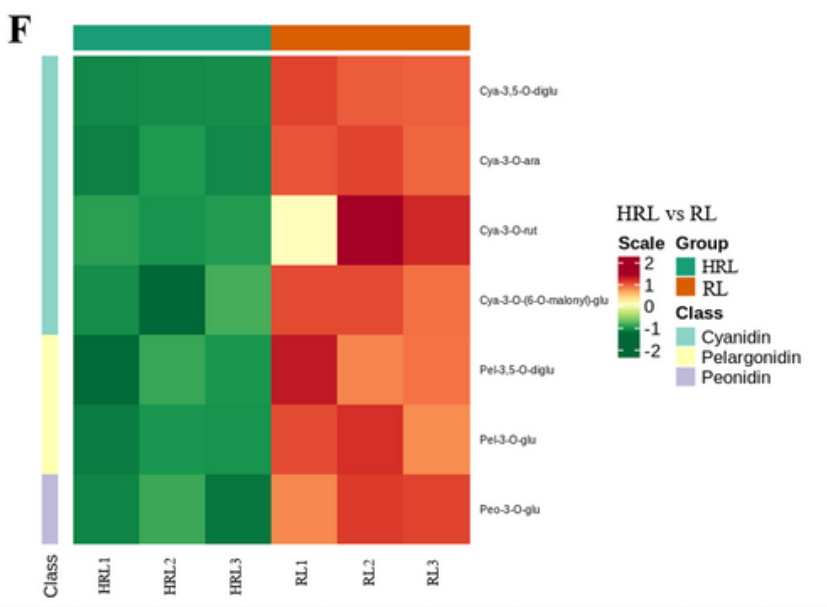

Figure 2 
Metabolite expression level analysis of A. mandshuricum in different leaf sample groups. (A) The histogram of antho-cyanidin levels of three leaf samples. (B) Anthocyanidin expression level of different groups. (C-F) Differentially accumulated metabolites (DAMs) in the anthocyanin biosynthesis pathway between four comparative groups of samples of A. mandshuricum. These DAMs were analyzed by KEGG enrichment analysis (C, GL vs HRL; D, GL vs HRL_RL; E, GL vs RL; F, HRL vs RL). (G) Venn diagram of metabolites between three compared groups of leaf samples.

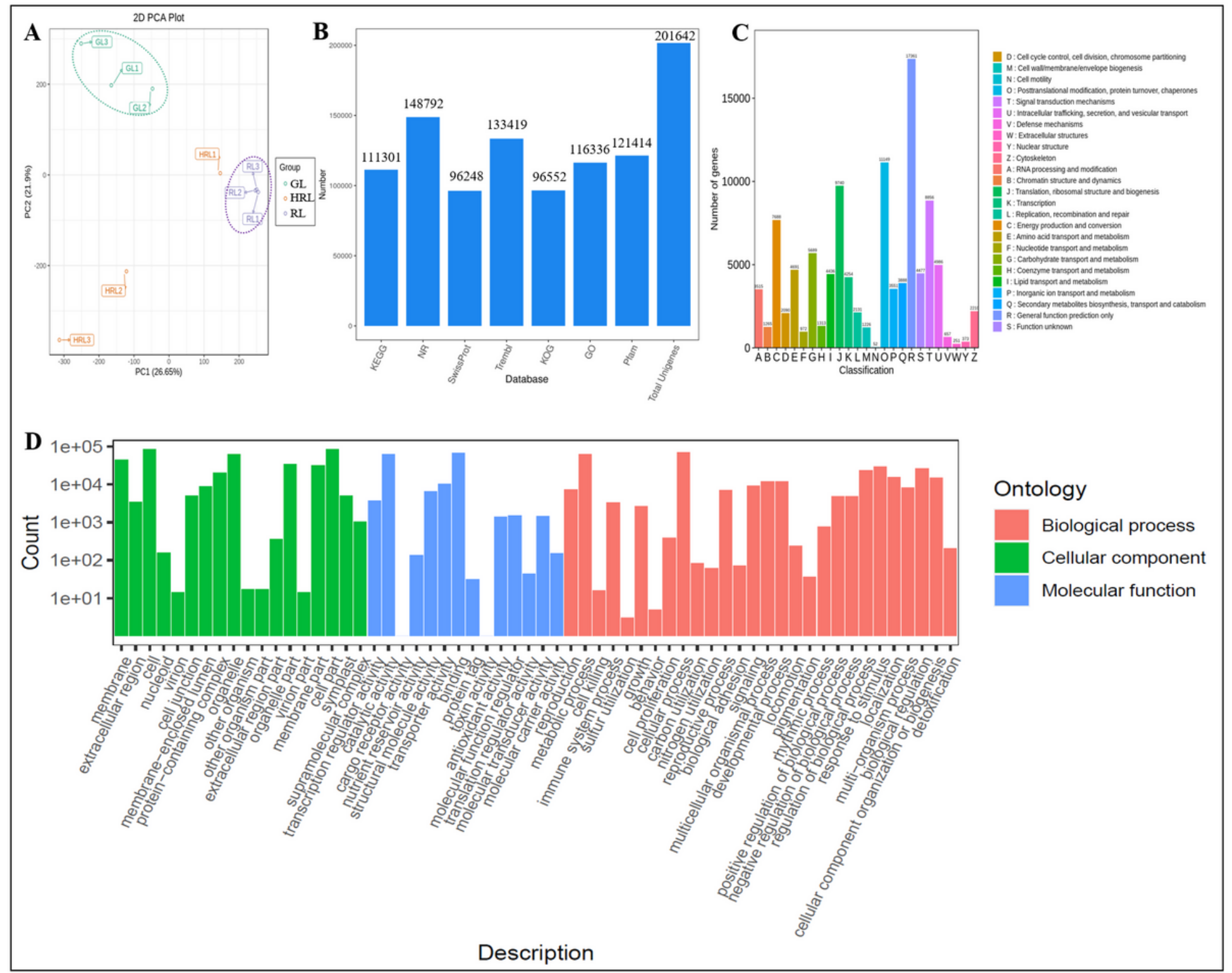

\section{Figure 3}

The principal component analysis and functional annotation of unigenes in A. mandshuricum. (A) Principal component analysis (PCA). (B) The histogram shows the number of unigenes annotated by BLAST in each database. (C) KOG classification of A. mandshuricum transcripts. (D) GO classification of A. mandshuricum transcripts. 

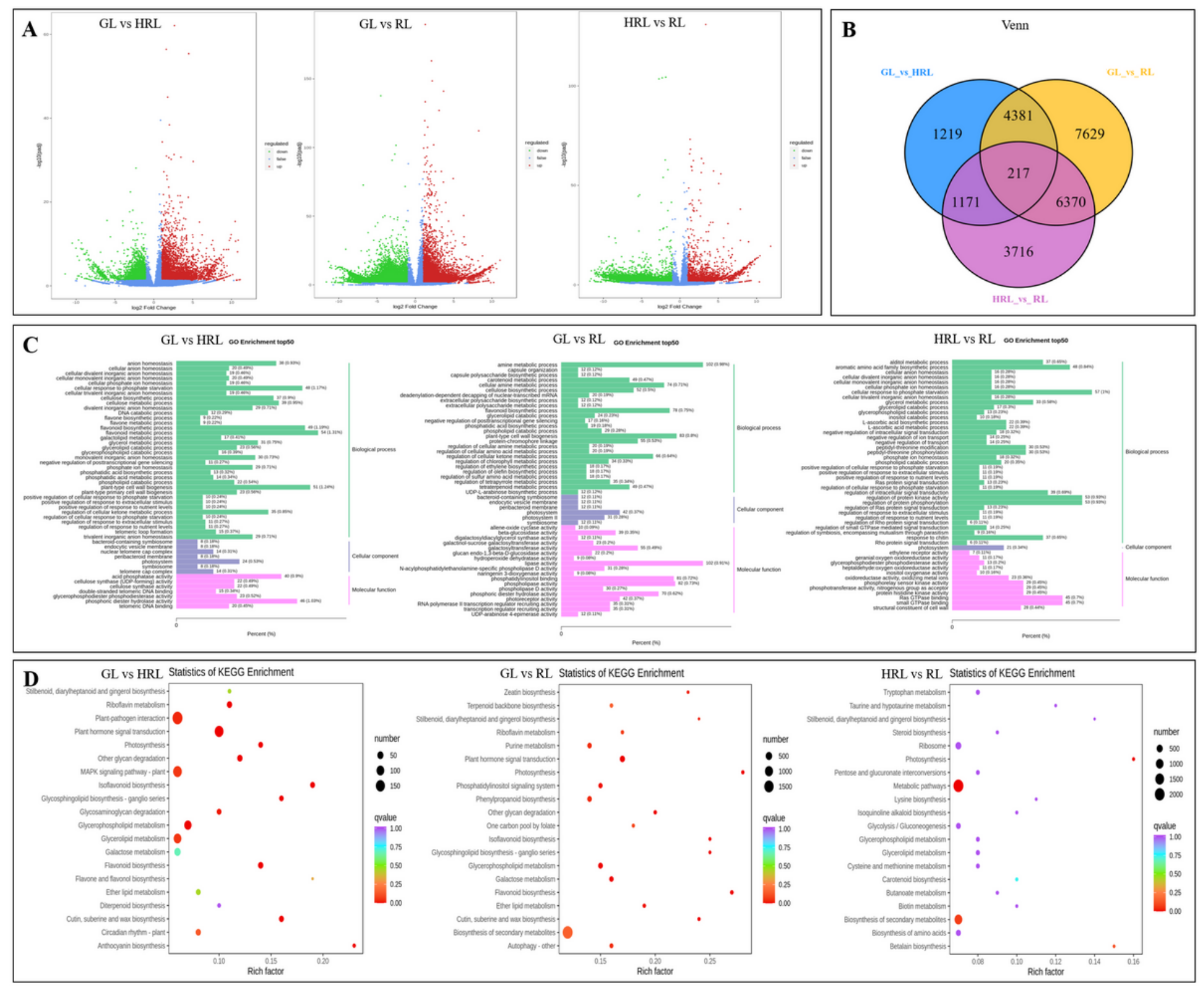

\section{Figure 4}

Differentially expressed genes between different colored leaves of A. mandshuricum. (A-C) Volcano plots were used to display the down-regulated, no-regulated and up-regulated genes between GL vs HRL, GL vs $\mathrm{RL}$ and HRL vs RL. (D) Venn map showing the common and respective DEGs between the three compared groups of leaf samples. 
A
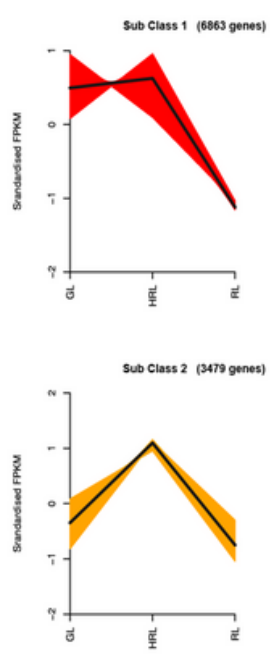
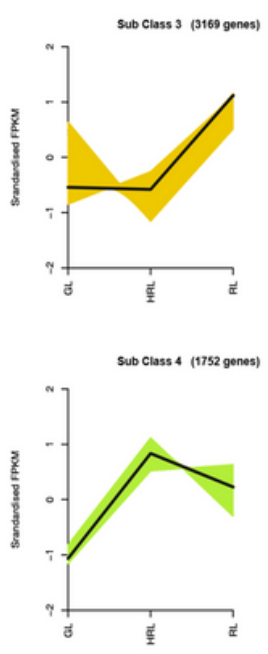

Sub Clisss 5 (5541 gones)
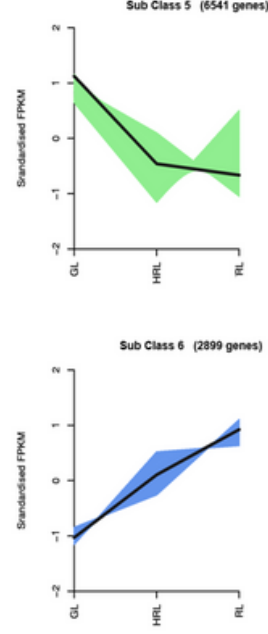

B

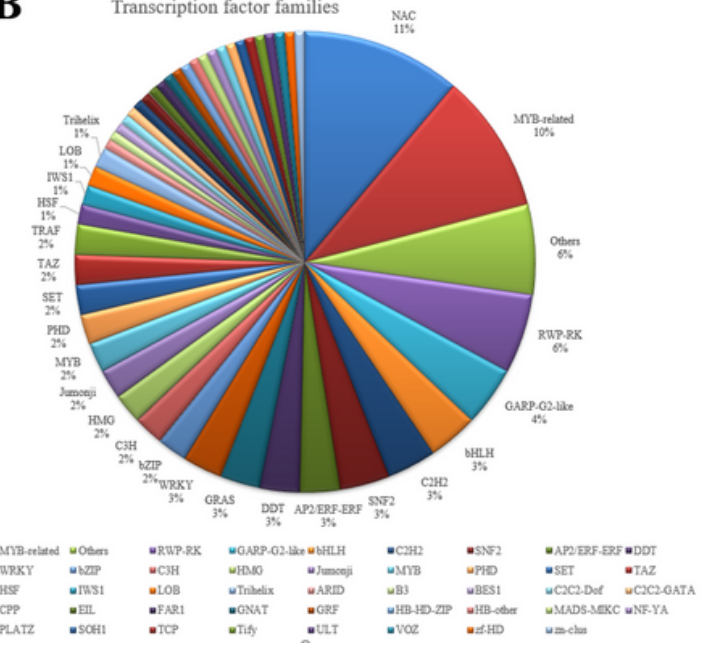

Figure 5

Six classes of co-expressed genes and their kinetic patterns. (A) Six classes of co-expressed genes. (B) Transcription factors (TFs) were encoded by these genes in class 6. 


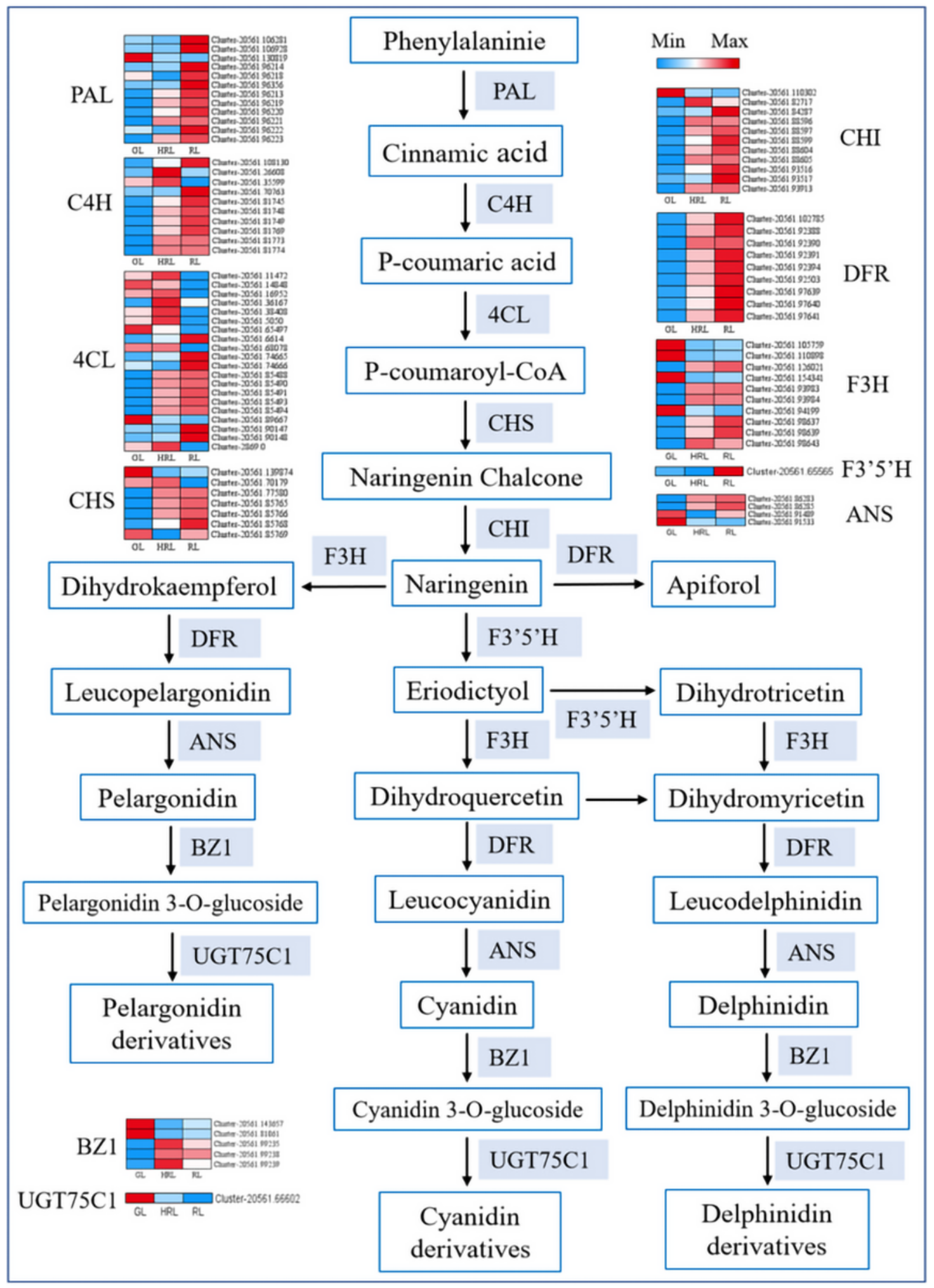

\section{Figure 6}

Modulation of phenylpropanoid, flavonoid, and anthocyanidin biosynthesis pathway genes during leaf color change in A. mandshuricum. Reconstruction of the phenylpropanoid, flavonoid and anthocyanidin biosynthetic pathways with differentially expressed structural genes. The heat map displays downregulated and up-regulated structural genes. 


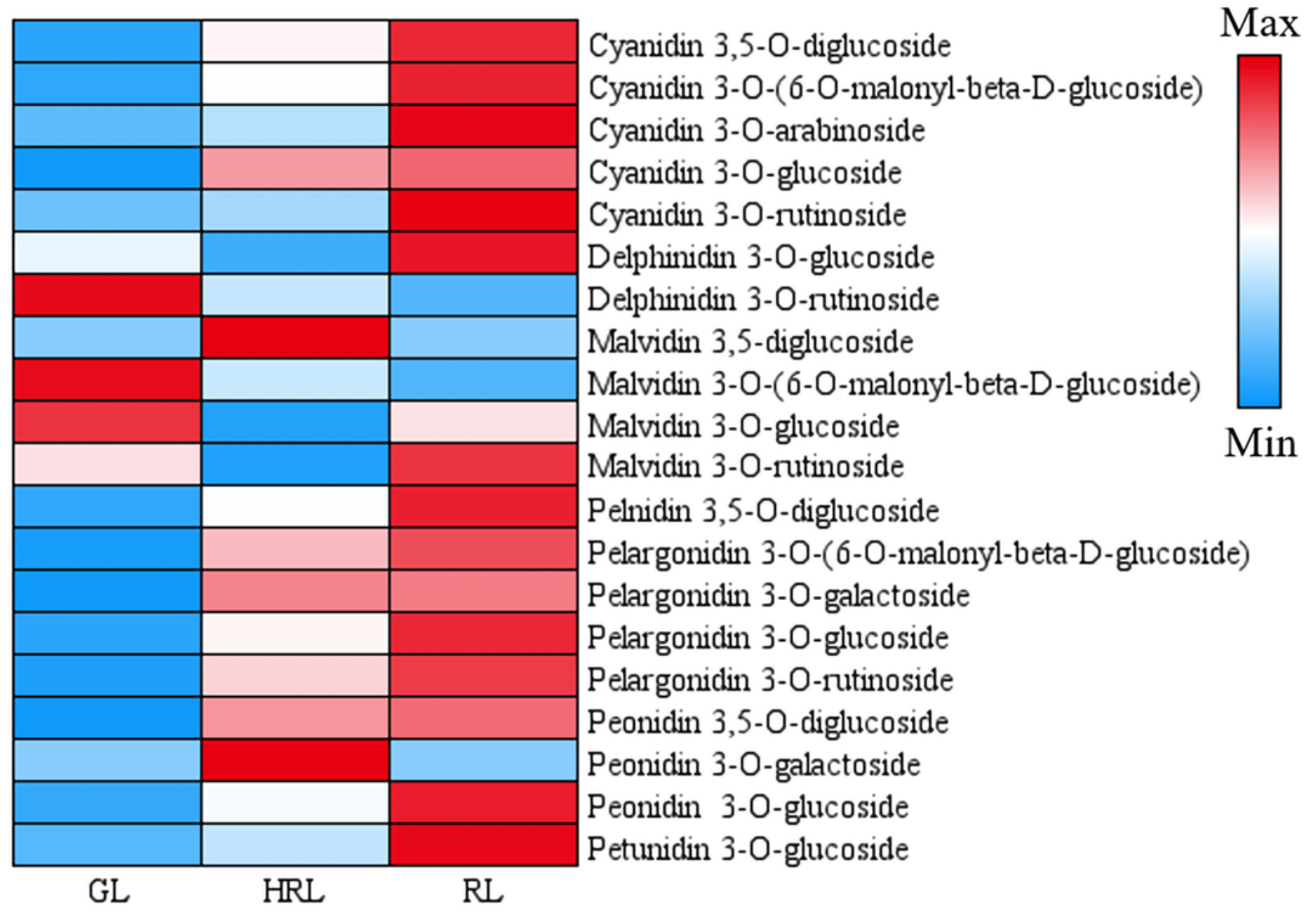

Figure 7

Metabolite accumulation level in anthocyanin synthesis pathways. 


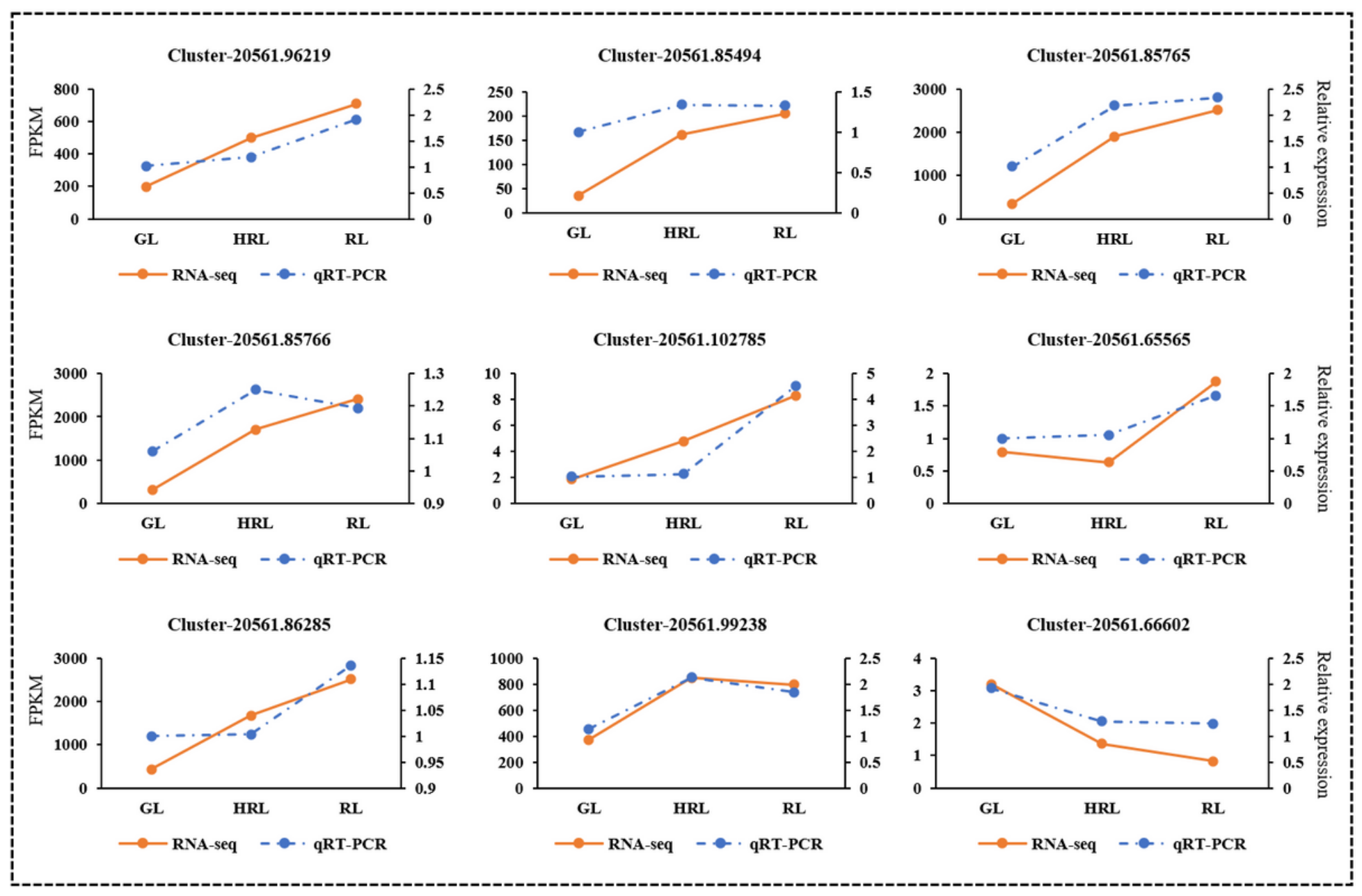

Figure 8

Verification of phenylpropanoid, flavonoid, and anthocyanidin biosynthesis-related differentially expressed genes (DEGs) by quantitative real-time polymerase chain reaction (qRT-PCR).

\section{Supplementary Files}

This is a list of supplementary files associated with this preprint. Click to download.

- FigureS1.docx

- TableS.zip 